\title{
Developing Motivational Visual Feedback for a New Telerehabilitation System for Motor Relearning after Stroke
}

\author{
Ekaterina Ivanova \\ Technische Universität Berlin \\ Industrial Automation Technology \\ ivanova@iwf.tu-berlin.de \\ Mareike Schrader \\ Charité Universitätsmedizin Berlin \\ Center for Stroke Research Berlin \\ mareike.schrader@charite.de
}

\author{
Katharina Lorenz \\ Universität der Künste \\ Design Research Lab \\ k.lorenz@udk-berlin.de
}

\author{
Michael Minge \\ Technische Universität Berlin \\ Cognitive Psychology and Cognitive Ergonomics \\ michael.minge@tu.berlin.de
}

\begin{abstract}
Motor relearning after stroke is a lengthy process which should be continued after patients get discharged from the clinic. This project aims at developing a system for telerehabilitation which enables stroke patients to exercise at home autonomously or under remote supervision of a therapist. The system includes haptic therapy devices which are more promising and beneficial for stroke rehabilitation than non-haptic approaches. In this paper, we present the results of two initial studies investigating specific design solutions for the task's and feedback's visualization. In the first study, we followed a participatory design approach to create a set of design solutions for a motivating instant visual feedback for exercising with the haptic devices. In the second study we evaluated the developed visualizations with stroke patients in clinical environment. The current project status and next steps are described.
\end{abstract}

Human-machine interaction; usability; rehabilitation robotics; telerehabilitation; stroke; gameful design.

\section{INTRODUCTION}

Strokes are among the leading causes for acquired disability (Johnston et al., 2009), as well as for deaths worldwide (Murray \& Lopez, 1997). Motor rehabilitation aims to help stroke patients in recovering voluntary motor movement capability, so that they can return to an independent lifestyle again. It is a lengthy process which can take months, sometimes years. In the rehabilitation clinic, patients receive an intensive therapy program on a day to day basis. After being discharged from the clinic, severely affected patients still need to continue this training to recover their motor abilities. However, this happens only rarely as treatments for follow-up rehabilitation at home are sparse. Deterioration of ADL (Activities of Daily Living) and of motor functions in general is the consequence (Langhammer \& Stanghelle, 2003). A possible solution is an individualized and motivating telerehabilitation system which can be used at home. Especially for the rehabilitation after a stroke, training at home is beneficial (Butler et al., 2014; Langhorne et al., 2011) and highly accepted by patients and health professionals (Johansson \& Wild, 2011).

Therapeutic robot-based devices are a promising direction in the development of effective telerehabilitation, as they allow the integration of haptic feedback. Robot-based rehabilitation is often applied in hospitals alongside conventional therapy and is beneficial for motor recovery after a stroke (Kwakkel et al., 2008; Norouzi-Gheidari et al., 2012). Several systems, including audio, video, and haptic modalities, already exist (Theriault et al., 2014; Weiss et al., 2014). However, most of them are limited to the use of low cost, commercial haptic devices for therapy training, such as joysticks. It can be assumed that devices specifically developed for stroke rehabilitation are more effective and beneficial for motor relearning (Langhammer \& Stanghelle, 2003; Norouzi-Gheidari et al., 2012). Making these devices available for home use could lead to more pronounced results than already existing low-cost solutions.

Since patients will train with the system at home independently, a key to the success of such systems 
is to ensure an appropriate feedback for patients. In addition, the patient's motivation has a great impact on rehabilitation progress. Being motivated is especially important for therapy at home without being supervised constantly by a clinical team. In this paper, we present the user-centred design and evaluation of visualizations of tasks and feedback. But first of all, we would like to introduce the planned robot-based telesystem for motor relearning in more detail.

\section{CONCEPT OF A ROBOT-BASED SYSTEM}

\subsection{Haptic Devices for Stroke Rehabilitation}

Two haptic devices which were specifically developed for therapy after stroke are Reha-Slide and Bi-Manu-Track (see Figure 1) and are currently used in clinical settings. Reha-Slide is designed for unilateral or bilateral training of the shoulder, elbow, and flexion or extension of the wrist (Hesse et al., 2008). With Reha-Slide, square movements in clockwise or counter clockwise direction can be exercised. Bi-Manu-Track can be used for training of bimanual pronation and supination movement of the forearm and flexion and extension of the wrist in parallel or symmetric fashion (Hesse et al., 2003). These aforementioned systems were the basis for the implementation of the robot based telerehabilitation in the current study.

\subsection{Robot-based telerehabilitation system}

The concept of the new telesystem has been presented in a previous research paper (Ivanova et al., 2015). A design overview is illustrated in Figure 2. The system consists of two work stations: a user interface for the patient (purple) and an interface for the therapist (green). Both interfaces comprise software and hardware. Two operation modes allow patients to train with the system. Patients can use the system either autonomously for individual training with the haptic device (autonomous mode) or for a one-on-one session with a therapist or a doctor (telesupervision mode).
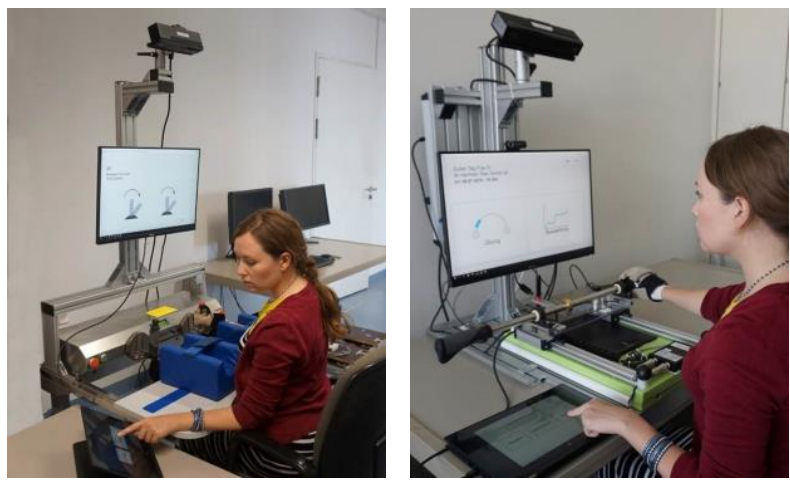

Figure 1: First prototype of telerehabilitation systems based on haptic therapeutic devices Bi-Manu-Track (left) and Reha-Slide (right).

In telesupervision mode, the therapist and the patient communicate with each other using audio, video, and haptic channels. When the patient exercises autonomously, therapy-relevant data is logged by the system. This information enables therapists to analyze previous training sessions and to adapt the further rehabilitation process to the individual needs and capabilities of the patient.

\subsection{Challenges from the user's point of view}

A major design challenge of the new robot-based telerehabilitation system is the implementation of a user interface that is both usable and motivating for the patient. Most stroke patients suffer from cognitive limitations in addition to physical and functional deficits (O'Sullivan \& Schmitz, 2007). For this reason, an intuitive and clear navigation concept is needed. Motivation is a further factor which has a great impact on the rehabilitation process (O'Sullivan \& Schmitz, 2007). This becomes especially important in home-based rehabilitation settings. One way to enhance motivation is to provide a gameful training environment and to include gamification elements. This approach has been successfully implemented in clinical settings (Burke et al., 2009) and could also be beneficial for home training (Johnson \& Schmidt, 2011). At the same time, systems which provide visual feedback

\section{A Autonomous Mode}

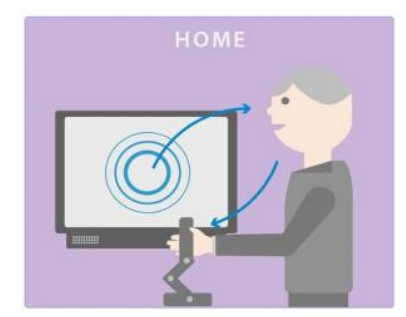

Haptic telerehabilitation device (patient station)

\section{B Telesupervision Mode}

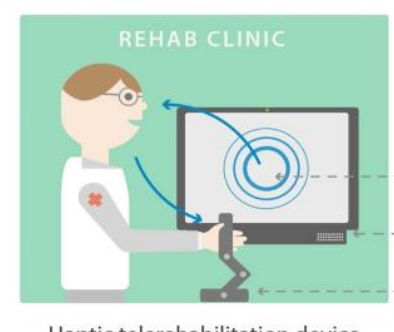

Haptic telerehabilitation device (therapist station)

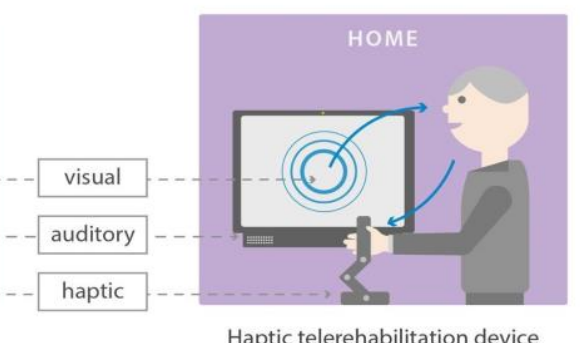

(patient station)

Figure 2: Concept of the telesystem for stroke rehabilitation. A: Autonomous mode (left), B: Telesupervision mode (right). 
are more beneficial for motor learning processes compared to systems without visual feedback (Laver et al., 2012). Therefore, it seems promising to develop a gameful system that provides the patient with therapeutic visual feedback about his or her movements.

\section{FINDING VISUAL FEEDBACK SOLUTIONS}

Using games or game elements in non-gaming contexts is a promising way to create engaging interfaces and to reinforce patients' motivation for therapy exercises. In particular, if patients continue rehabilitation at home, visual feedback supports them in exercising correctly and motivates them to practice on a regular basis.

To develop scenarios implementing motivational strategies by the use of visual feedback, a participatory design workshop was conducted (Sanders et al., 2011). To meet patients' needs and therapy requirements, it was essential to involve not only indirect users (i.e., three therapists) in a first step, but also project members with diverse expertise (i.e., a software developer, one engineer, one designer and two psychologists).

\subsection{Procedure}

In the beginning, the therapists demonstrated training movements with Reha-Slide and Bi-ManuTrack. The following requirements for the execution of movements were explained: (1) Range of motion, (2) symmetry, (3) smoothness, (4) tempo, and (5) fine motor skills. Before generating design solutions for visual feedback, assessment criteria for rating the ideas were discussed, defined, and prioritized. In the ideation session, participants used the provided material, such as inspirational pictures, sketches of games, paper and pencils to develop ideas for visual feedback (Figure 3). The ideas were then presented and rated. The most promising concepts were selected for implementation in order to be evaluated and optimized by patients as direct users in the next step.

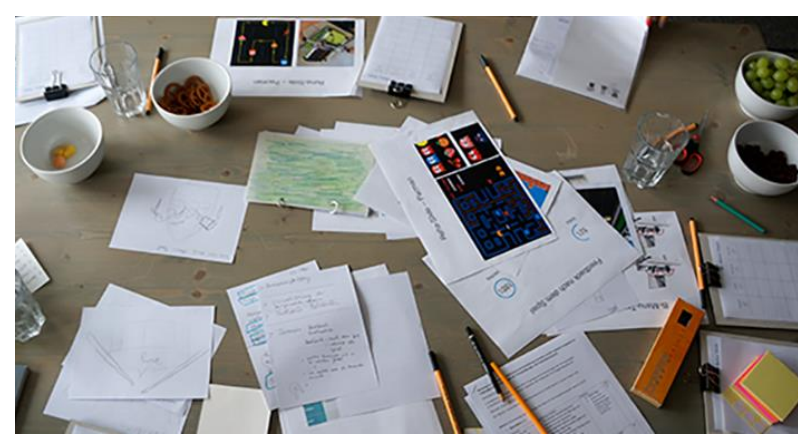

Figure 3: Participatory Design Workshop: Material that could be used in the ideation session.
The outcome of the workshop was a collection of instant feedback visualizations for both therapy devices, Reha-Slide and Bi-Manu-Track, which were rated by all workshop participants using the following predefined criteria: (1) short-term motivation, (2) long-term motivation, (3) familiarity with the specific activity of daily life, (4) congruence between visualization and exercise movement, (5) ease of comprehension, and (6) adaptability to individual motor skills.

After the workshop, the visualizations with the highest scores were translated into interactive animations. Two examples for each device are presented in the following. All four concepts use metaphors from everyday life, with the aim to make it easier for the patients to understand the abstract movements which they should perform using the Reha-Slide or the Bi-Manu-Track.

"Hanging up laundry" is a visual feedback for RehaSlide supporting patients to exercise shoulders, elbows and wrists (Figure 4). Patients move the arms back to the laundry basket and forward to the clothesline in order to "hang up" the laundry on the washing line. The goal of each session is to empty the laundry basket. The range of motion can be adapted to the patient's needs by changing the distance between the washing line and the laundry basket. There is also the possibility to support different exercises as square movements in clockwise or counter clockwise direction by changing the position of the laundry basket.

"Collecting stars" is an alternative feedback visualization for Reha-Slide (Figure 4). Patients can train their arm functions on different levels by collecting the appearing stars. Additionally, visual attention is trained, since patients have to notice the stars peripherally. This environment can be easily adapted to any level of difficulty by modifying the positions of the stars and their rate of appearance. It is also possible to motivate special movements by showing several stars simultaneously and to make the patient follow specific movements.

"Wiping the aquarium window glass" was rated as the most appropriate visualization for Bi-Manu-Track. When exercising, patients control wipers by performing pronation and supination movements. In a therapy session, the task is to wipe the aquarium glass so that the fishes in the aquarium are visible (Figure 5). This visualization provides a subtle, instant feedback about the performance (i.e., symmetry, smoothness, and range of motion). For example, if dirt on the aquarium window glass is left, this indicates a lower movement of the patient.

"Driving away flies" is to train both flexion and extension of the wrist with Bi-Manu-Track. Patients have to protect their cake by moving their hands constantly to drive away the flies (Figure 5). If patients stop moving their hands, the flies will start

\subsection{Results}



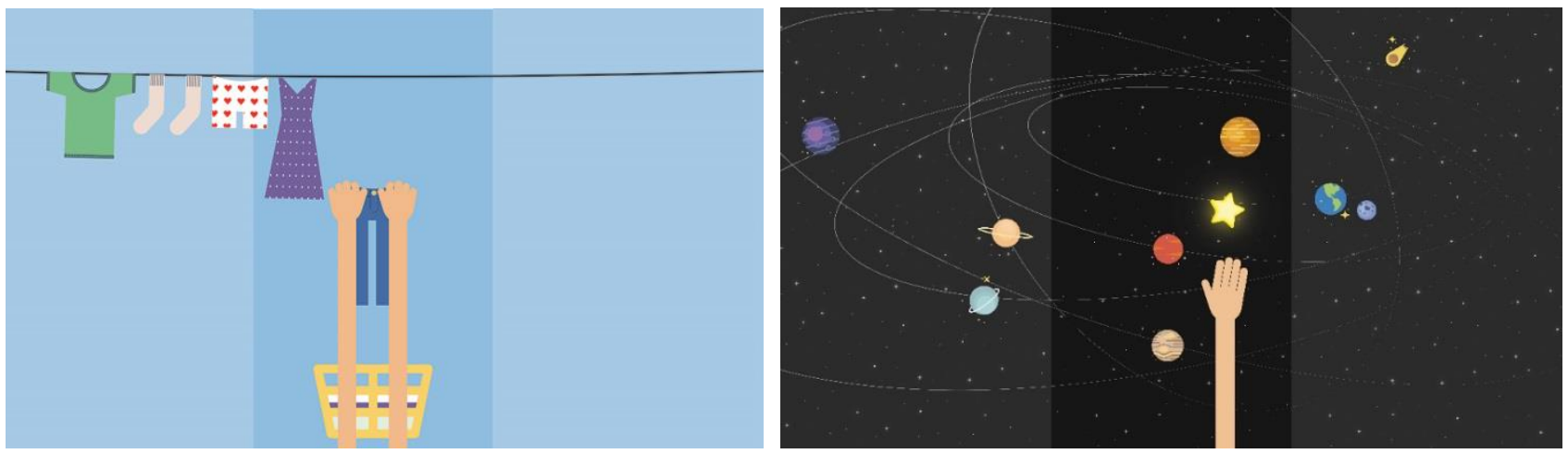

Figure 4: Visual feedback for Reha-Slide: Hanging up laundry (left) and Collecting stars (right).

eating the cake. The goal of this exercise is to keep as much of the cake as safe as possible.

In addition to the four gameful visualizations, we implemented two abstract feedback options for the Reha-Slide device. The goal of the abstract feedback options was to provide pragmatic, non-gamified visualizations, which may allow for a concentrated training with a particular movement goal. Examples for such exercises are the visualizations "Line" and "Rectangle". During these exercises, patients have to move along a defined trajectory (line or rectangle). The difficulty of the training can be manipulated through the width of the exercice area. In case of deviations from this area, the visualization changes the color and thereby indicates the movement error.

\section{EVALUATION OF VISUALIZATIONS FOR REHA-SLIDE}

After developing different concepts for visual feedback, four visualizations of patients' movements were evaluated: (1) Rectangle, (2) Line, (3) Collecting stars and (4) Hanging up Laundry. The main goal of the study was to determine patients' preferences and to explore whether they feel supported and encouraged to carry out the exercise.

\subsection{Participants}

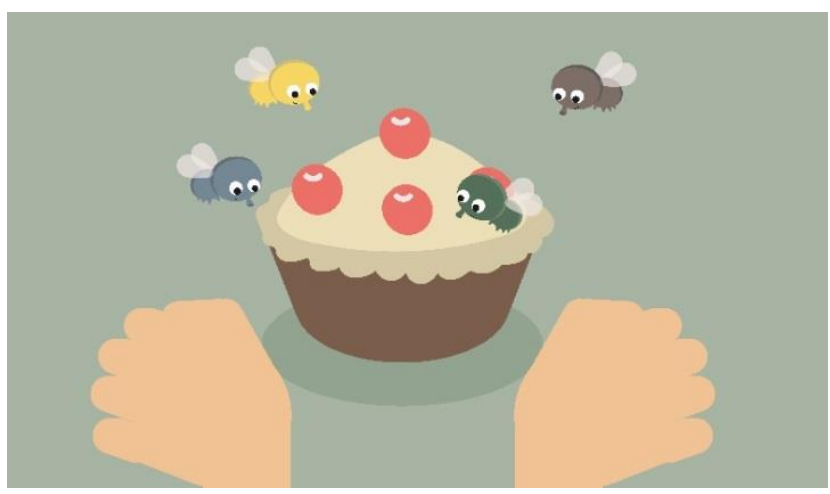

Six participants with neurological deficits ranging in age from 51 to 89 years $(M=71$ ys) took part in this study. All participants were patients of a neurological rehabilitation clinic. Four participants were male and two were female. Five patients suffered from a stroke and one from a hypoxic brain damage. Five patients were in the subacute phase, one patient was considered chronic. All participants showed deficits in the motor activity of the shoulder, arm and hand function. The patients were familiar to exercise with Reha-Slide.

\subsection{Method}

Each patient was given a short instruction about the procedure and the relevant tasks. Participants trained with all four feedback visualizations. The presentation order of the four exercises was counterbalanced over all participants. During the exercise „Rectangle“, the patient performed a sliding movement with both arms and brought the indicator in a clockwise movement around the rectangle. During the exercise "Line", the patient moved the indicator from bottom to top and back. In both exercises the indicator must be kept within the defined exercise area. If the indicator got out of the area a red sign was presented. In level 4 the exercise area was smaller and thus more difficult than in level 2. The patient started with level 2 and played each level for one minute. The goal in the game "Collecting stars" was to catch each flashing star with the virtual hand while moving the handles

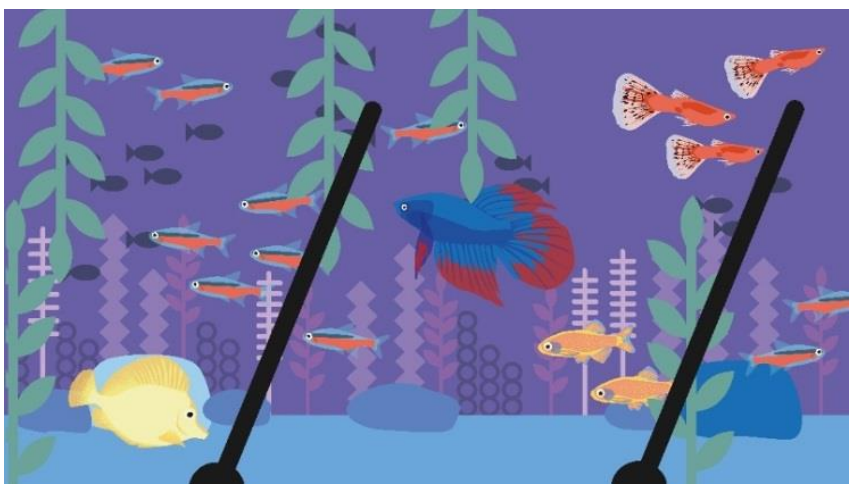

Figure 5: Visual feedback for Bi-Manu-Track: Driving away flies (left) and Wiping the aquarium window glass (right). 
up, sideways and down. During the game "Hanging up laundry", the patient should move the virtual arms to take the laundry out of the basket and hang it on the washing line. There was a shining marker where the clothes should be placed. In that way the patient not only performed an up-and-down movement but also moved the arms to the side. At the end of each exercise, the patients were interviewed with a questionnaire. The closed-ended questions were evaluated using a 5-point Likert scale (1 = "totally agree" to $5=$ "totally disagree").

\subsection{Results}

Overall almost $60 \%$ of the participants "totally agreed" that the visualizations helped them to increase their motivation and to put more effort into the exercise. Additionally, $20 \%$ of the patients "agreed" in that point. When asked about their concentration ability during the exercises, $71 \%$ of the participants reported that they could "concentrate very well" on the task. This result is underpinned with $75 \%$ of the patients replying that they felt "not at all distracted" by the motion visualization. Almost all patients $(95 \%)$ could see all of the graphical representations without any problems. Also, the patients were asked, if they think they will be bored with the exercise in the length of time. There was no uniform tendency reported and some differences between the exercises were revealed. All of the participations considered the Reha-Slide with visualization superior to the standalone system and over $80 \%$ of the interviewed participants classified the system as very helpful. Five of six patients could imagine to use the system even at home.

The patients were asked what they really liked about the visualization feature of the system. They answered frequently that the system increased their concentration and motivation and that they have to work more precisely. For improvement, they suggested to change the colour design of the "Rectangle" and "Line" exercises.

\section{Exercise I like most}

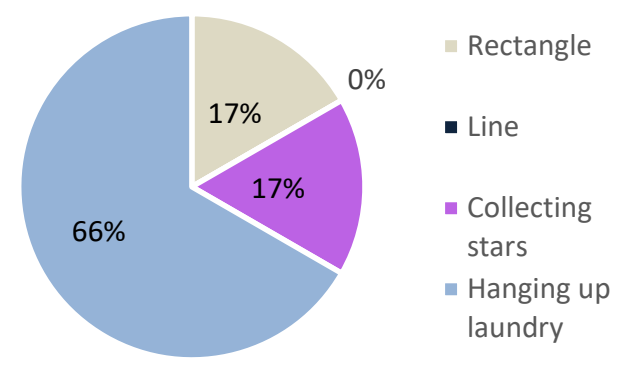

Figure 6. Participants' preferences for visual feedback.

After each exercise, the patients were requested to rate the visualizations (scale: 1 = "I like it very much" to 10 = "I do not like it at all"). The "Hanging up laundry" game received the best result $(M=2.2)$, followed by the "Collecting stars" game $(M=2.7)$. The "Line" and the "Rectangle" obtained an average rating $(M=3.2$ and $M=3.3)$. At the end the patients were asked which exercise they liked most of all (see figure 6).

The patients clearly preferred the game "Hanging up laundry" due to the motivational character and its relation to everyday life.

\section{CONCLUSION}

In this paper, we presented the results of a participatory design workshop creating motivational solutions for visual feedback of a new robot-based telesystem for stroke rehabilitation. Four feedback options for the Reha-slide device were evaluated with patients in a comparative user study.

The motion visualizations were highly accepted by all patients. They classified the new features as very meaningful and helpful. Especially the two exercises embedded in games increased the motivation of the participants. Due to the little number of interviewed patients, the results can only be interpreted with caution and not generalized. Further evaluation with a bigger number of participants is required.

Based on the findings, the telesystem for stroke rehabilitation using haptic therapeutic devices is currently being implemented into full functionality. As it is essential to validate our results in the following steps, stroke patients and therapists will participate continuously in the development to iteratively evaluate and optimize the implementation of the system. Three main research questions remain to be addressed in the following: (1) Testing the usability of the user interface in real home settings, (2) Evaluating motivational instant feedback in short-term and long-term use, and (3) Verifying the system's effectiveness in clinical studies.

\section{ACKNOWLEDGMENT}

We thank the Center for Stroke Research Berlin at Charité Universitätsmedizin for clinical support. Authors are thankful to Ilona Schubert for technical support and Natalie Jankowski for providing helpful comments on questionnaire.

\section{REFERENCES}

Burke, J.W., McNeill, M.D.J., Charles, D.K., Morrow, P.J., Crosbie, J.H., McDonough, S.M. (2009). Serious games for upper limb rehabilitation following stroke. Conference on Games \& Virtual Worlds for Serious Applications - VSGAMES'09, 103-110.

Butler, A.J., Bay, C., Wu, D., Richards, K.M., Buchanan, S. (2014). Expanding 
telerehabilitation of stroke through in-home robotassisted therapy. International Journal of Physical Medical Rehabilitation, 2(184), 2.

Hesse, S., Schulte-Tigges, G., Konrad, M., Bardeleben, K., Werner, C. (2003). Robotassisted arm trainer for the passive and active practice of bilateral forearm and wrist movements in hemiparetic subjects. Archives of physical medicine and rehabilitation, 84(6), 915-920.

Hesse, S., Werner, C., Pohl, M., Mehrholz, J., Puzich, U., Krebs, H.I. (2008). Mechanical arm trainer for the treatment of the severely affected arm after a stroke. American Journal of Physical Medicine \& Rehabilitation, 87(10), 779-788.

Ivanova, E., Krüger, J., Steingräber, R., Schmid, S., Schmidt, S., Hesse, S. (2015). Design and concept of a haptic robotic telerehabilitation system for upper limb movement training after stroke. International Conference on Rehabilitaton Robotics - ICORR, 666-671.

Ivanova, E., Minge, M., Schmidt, H., Thüring, M., Krüger, J. (2017). User-centered design of a patient's work station for haptic robot-based telerehabilitation after stroke Current Directions in Biomedical Engineering, 3, 39-43.

Johansson, T., Wild, C. (2011). Telerehabilitation in stroke care - a systematic review. Journal of Telemedicine and Telecare, 17(1), 1-6.

Johnson, M.J., Schmidt, H. (2011). Robot assisted neurological rehabilitation at home: Motivational aspects and concepts for tele-rehabilitation. Public Health Forum, 17(4), 8-11.

Johnston, S.C., Mendis, S., Mathers. C.D. (2009). Global variation in stroke burden and mortality: estimates from monitoring, surveillance, and modeling. The Lancet Neurology, 8(4), 345-354.

Kwakkel, G., Kollen, B.J., Krebs, H.I. (2008). Effects of robot-assisted therapy on upper limb recovery after stroke: a systematic review. Journal of Neurorehabilitation and Neural Repair, 22(2), 111-121.

Langhammer, B., Stanghelle, J.K. (2003). Bobath or motor relearning programme? A follow-up four years post stroke. Clinical Rehabilitation, 17(7), 731-734.

Langhorne, P., Bernhardt, J., Kwakkel, G. (2011). Stroke rehabilitation. The Lancet, 377(9778), 1693-1702.

Laver, K., George, S., Thomas, S., Deutsch, J.E., Crotty, M. (2012). Cochrane review: virtual reality for stroke rehabilitation. European Journal of physical and rehabilitation medicine, 48(3), 523530.

Murray, C.J.L., Lopez, A.D. (1997). Mortality by cause for eight regions of the world: Global
Burden of Disease Study. The Lancet, 349(9061), 1269-1276.

Norouzi-Gheidari, N., Archambault, P.S., Fung, J. (2012). Effects of robot-assisted therapy on stroke rehabilitation in upper limbs: systematic review and meta-analysis. Journal of Rehabilitation Research and Development, 49, 479-496.

O'Sullivan, S.B., Schmitz., T. (2007). Physical rehabilitation (5th Ed.). Philadelphia: F.A. Davis.

Sanders, E.B.N., Brandt, E., Binder, T. (2011). A framework for organizing the tools and techniques of participatory design. Proceedings of the 11th Participatory Design Conference. NY: ACM Press, 195-198.

Theriault, A., Nagurka, M., Johnson, M.J. (2014). Therapeutic potential of haptic TheraDrive: An affordable robot/computer system for motivating stroke rehabilitation. Proceedings of the5th International Conference on Biomedical Robotics and Biomechatronics, 415-420.

Weiss, P., Heldmann, M., Gabrecht, A., Schweikard, A., Münte, T.M., Maehle, T.E., (2014). A low cost tele-rehabilitation device for training of wrist and finger functions after stroke. Proceedings of the 8th International Conference on Pervasive Computer Technology for Healthcare, 422-425. 